\section{Dr J. H. Wildhaber est le premier lauréat du "Swiss Medical Weekly Young Investigator's Award»}

25000.- fr. pour la recherche: non seulement une excellente idée mais aussi un apport financier substantiel. Le comité scientifique du Journal Suisse de Médecine (JSM) lança l'idée, la direction des Editions médicales suisse la soutint et la réalisa! Un grand merci au JSM. Ceci est vraiment de bonne augure pour les jeunes chercheurs suisses: être reconnu pour le travail scientifique réalisé dans son propre pays.

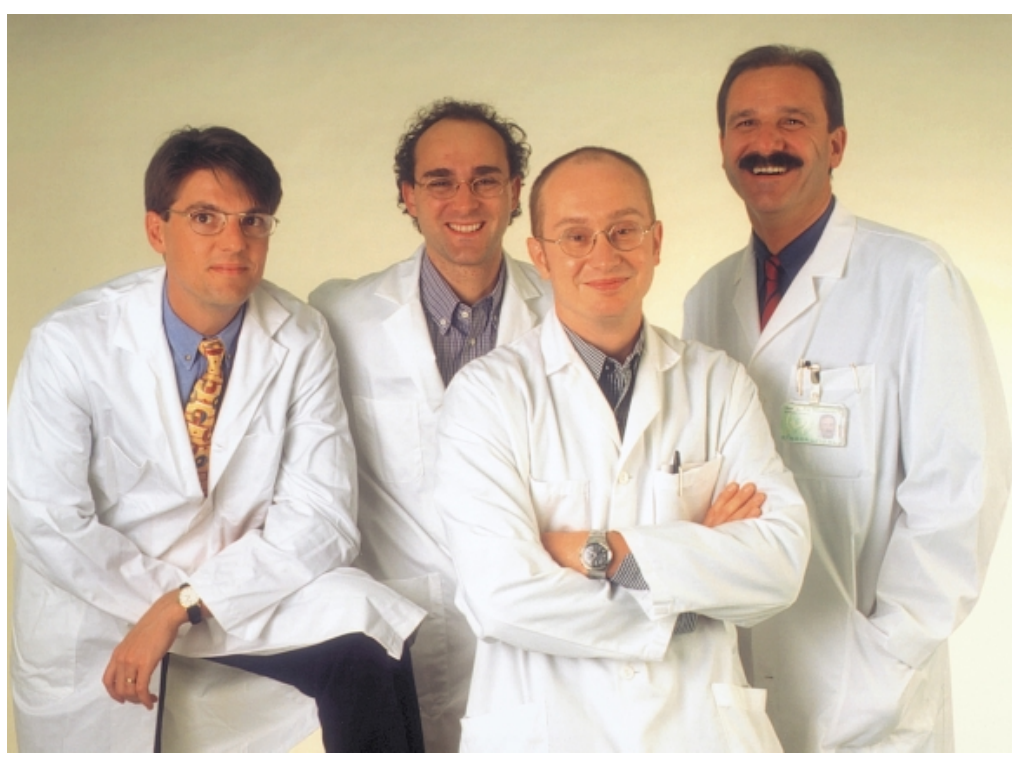

Groupe de recherche pneumologique à l'Hôpital universitaire d'enfants Zurich: G. L. Hall, A. Möller, J. H. Wildhaber, F. H. Sennhauser (de gauche à droite)

Publication originale: Schweiz Med Wochenschr 2000;130:529-34. Internet: www.smw.ch/archive/2000/130-15-374-00.html

Texte en totalité en format pdf sur www.smw.ch/pdf/2000_15/2000-15-374.pdf
Le premier lauréat $d u$ "Swiss Medical Weekly Young Investigator's Award" est le Docteur Johannes H. Wildhaber de l'Hôpital Pédiatrique Universitaire de Zurich. Nous remercions vivement tous les participants. Quinze manuscrits ont été soumis au concours. Peu de travaux ont dû être refusés, la grande majorité était d'excellente qualité scientifique: études bien construites, idées originales, résultats d'importance clinique et scientifique indéniables: un miroir de la recherche scientifique bio-médicale actuelle en Suisse.

Le Dr J. Wildhaber obtient le prix du JSM pour son travail:

Levels of exhaled nitric oxide in recurrently wheezy infants are decreased following inhaled steroid therapy (Wildhaber JH, Möller A, Hall GL, Sennhauser FH, Stick SM)

Les auteurs montrent d'une part qu'il existe une corrélation entre le taux de monoxide d'azote NO exhalé et le degré d'inflammation bronchique et d'autre part que la concentration de NO exhalé après thérapie par stéroïde topique/inhalé diminue. Des résultats similaires ont été publiés chez des sujets adultes, les résultats du Dr Wildhaber sont par contre les premiers publiés dans une population d'enfants en bas âge. Cependant le côté novateur de ce travail réside en premier lieu dans le développement de nouvelles techniques permettant de monitorer ce processus dans cette population. L'étude a été effectuée à l'Hôpital Pédiatrique Universitaire de Zurich en collaboration étroite avec le «Princess Margaret Hospital for Children" à Perth en Australie, où le lauréat a travaillé trois ans en recherche clinique et fondamentale.

L'attribution du prix aura lieu le 29 juin 2000 dans le cadre du programme de formation continue de la Clinique Pédiatrique Universitaire de Zurich.

$\begin{array}{cl}\text { 16:30 } & \begin{array}{l}\text { Présentation de cas cliniques de l'Hôpital } \\ \text { Pédiatrique (Dr U. Lips) }\end{array} \\ \text { 17:20 } & \begin{array}{l}\text { Présentation du travail primé } \\ \text { (Dr J. Wildhaber) }\end{array} \\ \text { 17:40 } & \begin{array}{l}\text { Laudatio et remise du prix } \\ \text { (Prof. A. P. Perruchoud) }\end{array}\end{array}$

La rédaction du JSM et la maison d'édition EMH se réjouissent d'annoncer que le "Swiss Medical Weekly Young Investigator's Award" sera dorénavant attribué annuellement pour autant que d'excellents travaux de recherche soient soumis. Nous ne doutons pas que de jeunes collègues effectuent une recherche scientifique de qualité en Suisse. Nous nous réjouissons déjà de la prochaine édition: chercheurs, mettez-vous au travail!

Pour le comité scientifique JSM Prof. André P. Perruchoud 